\title{
ON AGE-SPACE STRUCTURE OF AN AUTOSOMAL DIPLOID POPULATION DYNAMICS MODEL
}

\author{
VLADAS SKAKAUSKAS \\ Vilnius University \\ Naugarduko 24, 2600 Vilnius, Lithuania \\ E-mail:vladas.skakauskas@maf.vu.lt
}

\begin{abstract}
We discuss an age-structured autosomal polylocal multiallelic diploid population dynamics deterministic model taking into account random mating of sexes, females' pregnancy and its dispersal in whole space. Dispersal mechanism is described by the diffusion one with constant dispersal moduli while the birth moduli depend on the spatial density of the total population with a time delay. It is assumed that the population consists of male, single (nonfertilized) female, and fertilized female subclasses. Using the method of the fundamental solution for the uniformly parabolic second-order differential operator with bounded Hölder continuous coefficients we prove the existence and uniqueness theorem for the classic solution of the Cauchy problem for this model. We analyze population's growth and decay, too. Mutation is not considered in this paper.
\end{abstract}

\section{INTRODUCTION}

In a recent paper [4] we have proposed a general deterministic model for an age-structured autosomal polylocal multiallelic diploid population dynamics taking into account random mating of sexes without formation of the permanent male-female pair, females' pregnancy, possible destruction of the fetus (abortion), and female sterility periods after abortion and delivery. The class of the population of the given genotype is divided into five components: one male and four female, the latter four being the single (nonfertilized) female, fertilized female, female from the sterility period after abortion, and female from the sterility one following delivery. Each sex has three age-grades: prereproductive, reproductive, and post-reproductive. It is assumed that for each sex the commencement of each grade as well as the duration of the gestation and females' sterility periods are independent of individuals or time. Observe that this model neglects mutation of genes. In the case of the simplified 
model where abortion and sterility period after delivery are neglected the unique global classic solvability of that model for an unlimited population has been proved.

In the paper [6] we considered the same simplified model as in [4] letting, in addition, the population to disperse in whole space with the dispersal mechanism described by the general linear elliptic differential operator of second order. In that model we did not let birth moduli to depend on either the density of the total population or, more generally, on the population itself.

In the present paper we are interested in the model in [6] with simplified both the dispersal mechanism and mating law, and generalized birth moduli. The simplification consists in replacing the dispersal mechanism of the model in [6] by the diffusion one with constant dispersal moduli, and not letting the fertilization rate to depend on the characteristic of the mated male. Generalizing the model in [6] we let birth moduli to depend on the spatial density of the total population with a time delay. The aim of this article is to prove the existence and uniqueness of a classic solution of the Cauchy problem for this model.

The plan for this paper is as follows. In Sect. 3 we formulate the problem. Sect.4 represents hypotheses and results. In Sect.5 we recall some results concerning the solvability and uniquenees of the Cauchy problem for the linear differential parabolic operator of second order with a parameter. Sect.6 is devoted to proving the solvability theorem.

\section{NOTATION}

We examine the population whose autosomal character is controlled by $l$ loci of a pair of homologous chromosomes and a gene of the $j$ th locus can be in any of $m_{j}$ alleles. Let us recall notations in [4]:

$$
s=\left(\begin{array}{lll}
s_{11} & \ldots & s_{1 l} \\
s_{21} & \ldots & s_{2 l}
\end{array}\right) \text { and } k=\left(\begin{array}{ccc}
k_{11} & \ldots & k_{1 l} \\
k_{21} & \ldots & k_{2 l}
\end{array}\right) \text {, where } s_{i j}, k_{i j}=\overline{1, m_{j}},
$$

$j=\overline{1, l}, i=1,2$ : the genotypes (the homologous pairs of chromosomes, where $s_{11} \cdots s_{1 l}, k_{11} \cdots k_{1 l}$ are paternal and $s_{21} \cdots s_{2 l}, k_{21} \cdots k_{2 l}$ maternal chromosomes) for a male and a female, respectively;

$\tau_{1}, \tau_{2}, \tau_{3}:$ the ages of male, female, and embryo, respectively;

$t$ : time;

$E^{m}$ : Euclidean space (habitat of population) of dimension $m$;

$x=\left(x_{1}, x_{2}, \ldots, x_{m}\right)$ : the spatial position in $E^{m}$;

$u_{1 s}\left(x, t, \tau_{1}\right)$ : the age-space density of males of the $s$ genotype at age $\tau_{1}$, location $x$ and time $t$;

$u_{2 k}\left(x, t, \tau_{2}\right)$ : the age-space density of single (nonfertilized) females of the $k$ genotype at age $\tau_{2}$, location $x$ and time $t$;

$u_{3 s k}\left(x, t, \tau_{1}, \tau_{2}, \tau_{3}\right):$ the age-space density of fertilized females of the $k$ genotype at age $\tau_{2}$, position $x$ and time $t$ whose embryo is at age $\tau_{3}$ and that were fertilized by males at age $\tau_{1}$ and of the $s$ genotype;

$p_{s k}\left(x, t, \tau_{1}, \tau_{2}\right)$ : the density of probability to become fertilized for a female 
from the pair formed of male of the $s$ genotype at age $\tau_{1}$ and female of the $k$ genotype at age $\tau_{2}$, location $x$ and time $t$;

$\nu_{1 s}\left(x, t, \tau_{1}\right), \nu_{2 k}\left(x, t, \tau_{2}\right)$ and $\nu_{3 s k}\left(x, t, \tau_{1}, \tau_{2}, \tau_{3}\right):$ the death rates of males, single and fertilized females of characteristics $\left(s, \tau_{1}\right),\left(k, \tau_{2}\right)$ and $\left(s, \tau_{1} ; k, \tau_{2}\right)$, respectively, at position $x$ and time $t$;

$X_{k}\left(x, t, \tau_{2}\right)$ : the gain density of single females of characteristic $\left(k, \tau_{2}\right)$ by the females which have had a delivery at position $x$ and time $t$;

$Y_{k}\left(x, t, \tau_{2}\right)$ : the loss rate of single females of characteristic $\left(k, \tau_{2}\right)$ due to conception at location $x$ and time $t$;

$\omega_{s k}^{i}\left(x, t, \tau_{1}, \tau_{2}\right)$ : the probability a zygote to be of genotype $i$ provided that the pair of parents had characteristic $\left(s, \tau_{1} ; k, \tau_{2}\right)$;

$\sigma_{1}=\left(\tau_{11}, \tau_{12}\right], 0<\tau_{11}<\tau_{12}<\infty$ : the female sexual activity interval, $\bar{\sigma}_{1}=\left[\tau_{11}, \tau_{12}\right]$

$\sigma_{3}=(0, T], 0<T<\infty$ : the female gestation interval, $\bar{\sigma}_{3}=[0, T]$;

$\sigma_{2}\left(\tau_{3}\right)=\left(\tau_{21}+\tau_{3}, \tau_{22}+\tau_{3}\right], 0<\tau_{21}<\tau_{22}<\infty, \bar{\sigma}_{2}\left(\tau_{3}\right)=\left[\tau_{21}+\tau_{3}, \tau_{22}+\tau_{3}\right]$

$\sigma_{2}(0), \sigma_{2}(T)$ : the female fertilization and reproduction (delivery) intervals, respectively;

$n(x, t)$ : the spatial density of the total population at location $x$ and time $t$; $n_{1}(x, t)$ : the spatial density of males with ages from $\sigma_{1}$ at location $x$ and time $t$;

$b_{1 s k}\left(x, t, \tau_{1}, \tau_{2}, n(x, t-T)\right)$ and $b_{2 s k}\left(x, t, \tau_{1}, \tau_{2}, n(x, t-T)\right):$ the average numbers of male and female offspring, respectively, produced at position $x$ and time $t$ by a fertilized female of characteristics $\left(s, \tau_{1} ; k, \tau_{2}\right), \tau_{3}=T$;

$u_{1 s}^{0}\left(x, \tau_{1}\right), u_{2 k}^{0}\left(x, \tau_{2}\right), u_{3 s k}^{0}\left(x, \tau_{1}, \tau_{2}, \tau_{3}\right)$ : the initial distributions;

$n_{0}(x, t)$ : the initial spatial density of the total population at location $x$ and time $t \in[-T, 0]$;

$$
\begin{aligned}
& \sigma=\sigma_{1} \times \sigma_{2}(T), \bar{\sigma}=\bar{\sigma}_{1} \times \bar{\sigma}_{2}(T), d \sigma=d \tau_{1} d \tau_{2} ; \\
& \tau_{2}^{0}=0, \tau_{2}^{1}=\tau_{21}, \tau_{2}^{2}=\min \left(\tau_{21}+T, \tau_{22}\right), \tau_{2}^{3}=\max \left(\tau_{21}+T, \tau_{22}\right) \text {, } \\
& \tau_{2}^{4}=\tau_{22}+T, \tau_{2}^{5}=\infty \text {; } \\
& I=(0, \infty), \bar{I}=[0, \infty), I_{4}=\left(\tau_{2}^{4}, \infty\right), I_{j}=\left(\tau_{2}^{j}, \tau_{2}^{j+1}\right], j=\overline{0,3} ; \\
& I^{*}=\left(0, t^{*}\right], \bar{I}^{*}=\left[0, t^{*}\right], t^{*}<\infty \text {; } \\
& Q^{1}=\left\{\left(x, t, \tau_{1}\right) \in E^{m} \times I \times I\right\}, \bar{Q}^{1}=E^{m} \times \bar{I} \times \bar{I} ; \\
& Q^{2}=\left\{\left(x, t, \tau_{2}\right) \in E^{m} \times I \times\left(I \backslash \bigcup_{j=1}^{4} \tau_{2}^{j}\right)\right\}, \bar{Q}^{2}=\bar{Q}^{1}=E^{m} \times \bar{I} \times \bar{I} ; \\
& Q^{3}=\left\{\left(x, t, \tau_{1}, \tau_{2}, \tau_{3}\right) \in E^{m} \times I \times \sigma_{1} \times \sigma_{2}\left(\tau_{3}\right) \times \sigma_{3}\right\}, \\
& \bar{Q}^{3}=E^{m} \times \bar{I} \times \bar{\sigma}_{1} \times \bar{\sigma}_{2}\left(\tau_{3}\right) \times \bar{\sigma}_{3} \text {; } \\
& {\left[\left.u_{2 k}\right|_{\tau_{2}=\tau_{2}^{j}}\right] \text { : the jump of the function } u_{2 k} \text { at the plane } \tau_{2}=\tau_{2}^{j} \text {; }} \\
& \widehat{D}_{1}=\partial / \partial t+\partial / \partial \tau_{1}, \widehat{D}_{2}=\partial / \partial t+\partial / \partial \tau_{2}, \widehat{D}_{3}=\widehat{D}_{2}+\partial / \partial \tau_{3} ; \\
& D_{1}=\sqrt{2} \tilde{D}_{1}, D_{2}=\sqrt{2} \tilde{D}_{2}, D_{3}=\sqrt{3} \tilde{D}_{3}
\end{aligned}
$$
teristics of the operator $\widehat{D}_{i}$;

$a_{1 s}, a_{2 k}, a_{3 s k}$ : the spatial dispersal moduli of males, single females, and fertilized females, respectively;

$\Delta$ : the Laplace operator; 
$L_{1 s}\left(x, t, \tau_{1}\right)=a_{1 s} \Delta-\nu_{1 s}\left(x, t, \tau_{1}\right)$;

$L_{2 k}\left(x, t, \tau_{2}\right)=a_{2 k} \Delta-\nu_{2 k}\left(x, t, \tau_{2}\right)$;

$L_{3 s k}\left(x, t, \tau_{1}, \tau_{2}, \tau_{3}\right)=a_{3 s k} \Delta-\nu_{3 s k}\left(x, t, \tau_{1}, \tau_{2}, \tau_{3}\right)$;

$L^{1}(\eta ; \Omega)$ : the Banach space of functions $f(\eta, \cdot)$ integrable on $\Omega$;

$C^{0}\left(E^{m} \times J_{1} \times \ldots \times J_{r}\right), J_{j}=\left(J_{j 1}, J_{j 2}\right), J_{j 1}<J_{j 2}<\infty, j=\overline{1, r}$ : the Banach space of uniformly bounded continuous in $E^{m} \times J_{1} \times \ldots \times J_{r}$ functions $f\left(x, \xi_{1}, \xi_{2}, \ldots, \xi_{r}\right)$;

$C^{\alpha, 0, \ldots, 0}\left(E^{m} \times J_{1} \times \ldots \times J_{r}\right)$ : the Banach space of functions $f\left(x, \xi_{1}, \xi_{2}, \ldots, \xi_{r}\right)$ belonging to $C^{0}\left(E^{m} \times J_{1} \times \ldots \times J_{r}\right)$, which are Hölder continuous in $\left(E^{m} \times J_{1} \times\right.$ $\left.\ldots \times J_{r}\right)$ with exponent $\alpha \in(0,1)$ in $x$ uniformly with respect to $\left(\xi_{1}, \xi_{2}, \ldots, \xi_{r}\right)$, i.e. having the finite Holder seminorm with respect to $x$ (see [2]);

The letters $s$ and $k$ will be used in this paper only for the notation of genotypes of male and female, respectively.

\section{STATEMENT OF THE PROBLEM}

In this paper we discuss a model consisting of the following nonlinear system of integrodifferential equations, for $u_{1 s}, u_{2 k}, u_{3 s k}$,

$$
\begin{aligned}
& \left(D_{1}-L_{1 s}\right) u_{1 s}=0 \text { in } Q^{1}, \\
& \left(D_{2}-\tilde{L}_{2 k}\right) u_{2 k}=X_{k}, \tilde{L}_{2 k}=L_{2 k}-Y_{k} \text { in } Q^{2}, \\
& Y_{k}=\left\{\begin{array}{l}
0, \tau_{2} \notin \sigma_{2}(0), \\
n_{1}^{-1} \sum_{s} \int_{\sigma_{1}} p_{s k} u_{1 s} d \tau_{1}, n_{1}=\sum_{s} \int_{\sigma_{1}} u_{1 s} d \tau_{1}, \tau_{2} \in \sigma_{2}(0),
\end{array}\right. \\
& X_{k}=\left\{\begin{array}{l}
0, \tau_{2} \notin \sigma_{2}(T), \\
\left.\sum_{s} \int_{\sigma_{1}} u_{3 s k}\right|_{\tau_{3}=T} d \tau_{1}, \tau_{2} \in \sigma_{2}(T),
\end{array}\right. \\
& \left(D_{3}-L_{3 s k}\right) u_{3 s k}=0 \text { in } Q^{3}
\end{aligned}
$$

supplemented with the conditions

$$
\begin{aligned}
&\left.u_{1 s}\right|_{t=0}= u_{1 s}^{0},\left.u_{2 k}\right|_{t=0}=u_{2 k}^{0} \text { in } E^{m} \times I, \\
&\left.u_{3 s k}\right|_{t=0}= u_{3 s k}^{0} \text { in } E^{m} \times \sigma_{1} \times \sigma_{2}\left(\tau_{3}\right) \times \sigma_{3}, \\
&\left.u_{1 s}\right|_{\tau_{1}=0}=\left.\sum_{i k} \int_{\sigma} b_{1 i k}(x, t, \cdot, n(x, t-T)) u_{3 i k}\right|_{\tau_{3}=T} \omega_{i k}^{s} d \sigma \text { in } E^{m} \times I, \\
&\left.u_{2 k}\right|_{\tau_{1}=0}=\left.\sum_{s i} \int_{\sigma} b_{2 s i}(x, t, \cdot, n(x, t-T)) u_{3 s i}\right|_{\tau_{3}=T} \omega_{s i}^{k} d \sigma \text { in } E^{m} \times I, \\
& n(x, t)=\left\{\begin{array}{l}
\sum_{s} \int_{I} u_{1 s} d \tau_{1}+\sum_{k} \int_{I} u_{2 k} d \tau_{2}+ \\
\sum_{s k} \int_{\sigma_{3}} d \tau_{3} \int_{\sigma_{2}\left(\tau_{3}\right)} d \tau_{2} \int_{\sigma_{1}} u_{3 s k} d \tau_{1} \text { in } E^{m} \times I, \\
n_{0}(x, t) \text { in } E^{m} \times[-T, 0],
\end{array}\right. \\
&\left.u_{3 s k}\right|_{\tau_{3}=0}=p_{s k} u_{1 s} u_{2 k} / n_{1} \text { in } E^{m} \times I \times \sigma_{1} \times \sigma_{2}(0),
\end{aligned}
$$




$$
\left[\left.u_{2 k}\right|_{\tau_{2}=\tau_{2}^{j}}\right]=0, j=\overline{1,4} \text { in } E^{m} \times I,
$$

and governs evolution of the population with dispersal in whole space. In addition, we assume that initial distributions $u_{1 s}^{0}, u_{2 k}^{0}, u_{3 s k}^{0}, n_{0}$ satisfy the following compatibility conditions

$$
\begin{aligned}
& \left.u_{1 s}^{0}\right|_{\tau_{1 s}=0}=\left.\left.\left.\sum_{i k} \int_{\sigma} b_{1 i k}\right|_{t=0} u_{3 i k}^{0}\right|_{\tau_{3}=T} \omega_{i k}^{s}\right|_{t=0} d \sigma \text { in } E^{m}, \\
& \left.u_{2 k}^{0}\right|_{\tau_{2 k}=0}=\left.\left.\left.\sum_{s i} \int_{\sigma} b_{2 s i}\right|_{t=0} u_{3 s i}^{0}\right|_{\tau_{3}=T} \omega_{s i}^{k}\right|_{t=0} d \sigma,\left[\left.u_{2 k}^{0}\right|_{\tau_{2}=\tau_{2}^{j}}\right]=0, \\
& l=\overline{1,4} \text { in } E^{m} \\
& \left.u_{3 s k}^{0}\right|_{\tau_{3}=0}=\left.p_{s k}\right|_{t=0} u_{1 s}^{0} u_{2 k}^{0} / \int_{\sigma_{1}} u_{1 s}^{0} d \tau_{1} \text { in } E^{m} \times \sigma_{1} \times \sigma_{2}(0) . \\
& \left.n_{0}\right|_{t=0}=\sum_{s} \int_{I} u_{1 s}^{0} d \tau_{1}+\sum_{k} \int_{I} u_{2 k}^{0} d \tau_{2}+ \\
& \sum_{s k} \int_{\sigma_{3}} d \tau_{3} \int_{\sigma_{2}\left(\tau_{3}\right)} d \tau_{2} \int_{\sigma_{1}} u_{3 s k}^{0} d \tau_{1} \text { in } E^{m} .
\end{aligned}
$$

As it follows from the foregoing given functions $\nu_{1 s}, \nu_{2 k}, \nu_{3 s k}, p_{s k}, b_{1 s k}$, $b_{2 s k}, u_{1 s}^{0}, u_{2 k}^{0}, u_{3 s k}^{0}, n_{0}, \omega_{i k}^{s}, \omega_{s i}^{k}$ and the unknown ones $u_{1 s}, u_{2 k}, u_{3 s k}$ must be positive-valued, otherwise they have no biological significance. Our purpose is to find $u_{1 s}, u_{2 k}, u_{3 s k}$ verifying (1)-(13).

Observe that, for one-locus Mendel's population, $\omega_{s k}^{i}=\frac{1}{4}\left(\delta_{s_{11}}^{i_{11}}+\delta_{s_{21}}^{i_{11}}\right)\left(\delta_{k_{11}}^{i_{21}}+\right.$ $\delta_{k_{21}}^{i_{21}}$ ), where $\delta_{\beta}^{\gamma}$ designates the Kronecker symbol (see [7]).

\section{HYPOTHESES AND RESULTS}

Unless otherwise stated, assumptions listed in this section hold throughout the paper:

$\left(H_{1}\right) p_{s k}=p_{k}\left(x, t, \tau_{2}\right) \in C^{\alpha, 0,0}\left(E^{m} \times \bar{I} \times \bar{\sigma}_{2}(0)\right)$ does not depend on the characteristic of the mated male and has the compact support in $x$ $\left(\operatorname{supp} p_{k}\left(\cdot, t, \tau_{2}\right)\right)$ for any set $\left(t, \tau_{2}\right)$;

$\left(H_{2}\right) \omega_{s k}^{i} \in C^{0}\left(E^{m} \times \bar{I} \times \bar{\sigma}_{1} \times \bar{\sigma}_{2}(T)\right), b_{1 s k}, b_{2 s k} \in C^{0}\left(E^{m} \times \bar{I} \times \bar{\sigma}_{1} \times \bar{\sigma}_{2}(T) \times \bar{I}\right)$ are nonnegative functions;

$\left(H_{3}\right) \nu_{1 s} \in C^{\alpha, 0,0}\left(\bar{Q}^{1}\right), \nu_{2 k} \in C^{\alpha, 0,0}\left(\bar{Q}^{2}\right), \nu_{3 s k} \in C^{\alpha, 0,0,0,0}\left(\bar{Q}^{3}\right)$ are nonnegative functions;

$\left(H_{4}\right) a_{1 s}, a_{2 k}, a_{3 s k}$ are positive constants;

$\left(H_{5}\right) u_{2 k}^{0}\left(x, \tau_{2}\right) \in C^{0}\left(E^{m} \times \bar{I}\right)$ is nonnegative integrable w.r.t. $\tau_{2} \in I$, $u_{3 s k}^{0}\left(x, \tau_{1}, \tau_{2}, \tau_{3}\right) \in C^{0}\left(E^{m} \times \bar{\sigma}_{1} \times \bar{\sigma}_{2}\left(\tau_{3}\right) \times \bar{\sigma}_{3}\right)$ and $n_{0}(x, t) \in C^{0}\left(E^{m} \times[-T, 0]\right)$ are nonnegative, $u_{1 s}^{0}\left(x, \tau_{1}\right) \in C^{0}\left(E^{m} \times \bar{I}\right)$ is strictly positive integrable w.r.t. $\tau_{1} \in I$, and all the densities $u_{1 s}^{0}, u_{2 k}^{0}, u_{3 s k}^{0}, n_{0}(x, 0)$ verify (13).

Now we list theorems for solvability of model (1)-(13), population growth and its decay. The first of them will be proved in Sect. 6 while proof of the other two theorems is the same as that in [6].

THEOREM 1. Under the hypotheses $\left(H_{1}\right)-\left(H_{5}\right)$ problem (1)-(12) has for any 
$I^{*}$ an unique nonnegative classic (see [2,3]) solution such that $u_{1 s} \in C^{0}\left(E^{m} \times\right.$ $\left.\bar{I}^{*} \times \bar{I}\right) \bigcap L^{1}(x, t ; I), u_{2 k} \in C^{0}\left(E^{m} \times \bar{I}^{*} \times \bar{I}\right) \bigcap L^{1}(x, t ; I), u_{3 s k} \in C^{0}\left(E^{m} \times\right.$ $\left.\bar{I}^{*} \times \bar{\sigma}_{1} \times \bar{\sigma}_{2}\left(\tau_{3}\right) \times \bar{\sigma}_{3}\right)$.

Let us introduce the following notions

$$
\begin{aligned}
& \widehat{b}=\max _{s, k}\left\{\sum_{j} \int_{\sigma_{2}(T)} \sup _{i, E^{m} \times \bar{I} \times \bar{\sigma}_{1} \times \bar{I}}\left(b_{1 i j} \omega_{i j}^{s}\right) d \tau_{2},\right. \\
& \left.\sum_{j} \int_{\sigma_{2}(T)} \sup _{\tilde{\nu}_{2}=E^{m} \times \bar{I} \times \bar{\sigma}_{1} \times \bar{I}}\left(b_{2 i j} \omega_{i j}^{k}\right) d \tau_{2}\right\}, \quad \widehat{p}=\sup _{k, E^{m} \times \bar{I} \times \bar{\sigma}_{2}(0)} p_{k}, \\
& \inf _{k, E^{m} \times \bar{I} \times\left(\bar{I}_{2} \cup \bar{I}_{3}\right)} \nu_{2 k}, \quad \widehat{u}=\max _{s, k}\left(\sup _{E^{m} \times \bar{I}} u_{1 s}^{0}, \sup _{E^{m} \times \bar{I}} u_{2 k}^{0}\right), \\
& \widehat{u}_{3}=\max _{s, k}\left(\left.\sum_{i} \sup _{E^{m} \times \bar{\sigma}_{2}(T)} \int u_{\sigma_{1}}^{0} u_{3 i k}\right|_{\tau_{3}=T^{\prime}} \omega_{i k}^{s} d \tau_{1},\right. \\
& \left.\left.\sum_{i} \sup _{E^{m} \times \bar{\sigma}_{2}(T)} \int_{\sigma_{1}} u_{3 s i}^{0}\right|_{\tau_{3}=T} \omega_{s i}^{k} d \tau_{1}\right), \\
& Q_{*}^{3}=\left\{\left(x, t, \tau_{1}, \tau_{2}, \tau_{3}\right): x \in E^{m}, 0<t \leq \tau_{3}, \tau_{1} \in \sigma_{1}, \tau_{2} \in \sigma_{2}\left(\tau_{3}\right), \tau_{3} \in \sigma_{3}\right\}, \\
& Q^{3 *}=\left\{\left(x, t, \tau_{1}, \tau_{2}, \tau_{3}\right): x \in E^{m}, t>\tau_{3}, \tau_{1} \in \sigma_{1}, \tau_{2} \in \sigma_{2}\left(\tau_{3}\right), \tau_{3} \in \sigma_{3}\right\} .
\end{aligned}
$$

Theorem 2. Assume $\left(H_{1}\right)-\left(H_{5}\right)$ hold, and let $\tilde{\nu}_{2}>0$. Then

$$
\text { (i) } \sum_{s} \int_{\sigma_{1}} u_{3 s k} d \tau_{1} \leq\left\{\begin{array}{l}
\widehat{u}_{3} \text { in } Q_{*}^{3} \\
\widehat{p} \sup _{y \in E^{m}} u_{2 k}\left(y, t-\tau_{3}, \tau_{2}-\tau_{3}\right) \text { in } Q^{3 *} \text { for } t-\tau_{3} \in \bar{I}^{*},
\end{array}\right.
$$

(ii) $u_{1 s}, u_{2 k} \leq \delta \gamma^{j} \widehat{u}$ for $t \in(j T,(j+1) T] \cap\left[0, t^{*}\right], x \in E^{m}, \tau_{1}, \tau_{2} \in \bar{I}$, where $j=0,1, \ldots, \gamma=\max \left(\widehat{b} \widehat{p}, 1, \widehat{p} / \tilde{\nu}_{2}\right)$ and $\delta=\max \left(\widehat{b} \widehat{u}_{3} / \widehat{u}, 1, \widehat{u}_{3} / \widehat{u} \tilde{\nu}_{2}\right)$, (or more roughly $u_{1 s}, u_{2 k} \leq \delta \widehat{u} \gamma^{t / T}$ ).

$$
\begin{aligned}
& \text { Define } \\
& q=\widehat{b} \widehat{u}_{3} / \widehat{u}, \tilde{\nu}=\min _{s, k}\left(\inf _{\bar{Q}_{1}} \nu_{1 s}, \inf _{\bar{Q}_{2}} \nu_{2 k}\right), \\
& \omega_{0}=\left\{(x, t, \xi): x \in E^{m}, 0 \leq t \leq \xi, \xi \in \bar{I}\right\}, \\
& \omega_{j}=\left\{(x, t, \xi): x \in E^{m},(j-1) \tau_{2}^{4}<t-\xi \leq j \tau_{2}^{4}, t \leq t^{*}, \xi \in \bar{I}\right\}, j=1,2, \ldots
\end{aligned}
$$

Theorem 3. Assume the hypotheses $\left(H_{1}\right)-\left(H_{5}\right)$ hold and let

$$
\widehat{b} \hat{p} \leq q \leq \min (1, \tilde{\nu} \widehat{b}), \tilde{\nu}>0 . \text { Then } \max _{s, k}\left\{\sup _{\left(x, t, \tau_{1}\right) \in \omega_{j}} u_{1 s}, \sup _{\left(x, t, \tau_{2}\right) \in \omega_{j}} u_{2 k}\right\} \leq
$$
$\widehat{u} q^{j}$.

Corollary 4. Let assumptions of Th.3 hold. If $q<1$, then the population vanishes as $t$ increases. 


\section{SOME PROPERTIES OF THE PARABOLIC OPERATOR OF SECOND ORDER}

In this section we collect some results concerning the solvability and uniqueness of the Cauchy problem for the linear differential parabolic operator of second order (see $[2,3,5])$ with a parameter.

Lemma 5. (see [5]). Let

$$
\Lambda(x, t, \beta)=\partial / \partial t-\sum_{i, j=1}^{m} b_{i j} \partial^{2} / \partial x_{i} \partial x_{j}-\sum_{i=1}^{m} \tilde{b}_{i} \partial / \partial x_{i}+\tilde{b}_{0}
$$

be a uniformly parabolic operator depending on a parameter $\beta \in J=\left[\beta_{1}, \beta_{2}\right]$, $\beta_{1}<\beta_{2}<\infty$ with coefficients verifying the following conditions

$$
\begin{aligned}
& b_{i j}(x, t, \beta) \in C^{\alpha, \alpha / 2,0}\left(E^{m} \times \bar{I}^{*} \times J\right), i, j=\overline{1, m}, \\
& \tilde{b}_{i}(x, t, \beta) \in C^{\alpha, 0,0}\left(E^{m} \times \bar{I}^{*} \times J\right), i=\overline{0, m}
\end{aligned}
$$

and assume that

$0<u^{0}(x, \beta) \in C^{0}\left(E^{m} \times J\right)$,

$0<f(x, t, \beta) \in C^{0}\left(E^{m} \times \bar{I}^{*} \times J\right),|f(x, t, \beta)-f(y, t, \beta)| \leq \kappa t^{-\gamma}|x-y|^{\alpha}$

with $\kappa$ a constant and $\gamma \in(0,1)$. Then the problem

$$
\Lambda u=f \text { in } E^{m} \times \bar{I}^{*} \times J, u(x, 0, \beta)=u^{0} \text { in } E^{m} \times J
$$

has a unique stictly positive in $E^{m} \times \bar{I}^{*} \times J$ classic (see [2]) solution

$$
\begin{gathered}
u(x, t, \beta)=\int_{E^{m}} \Gamma(x, t ; y, 0 ; \beta) u^{0}(y, \beta) d y \\
+\int_{0}^{t} d \tau \int_{E^{m}} \Gamma(x, t ; y, \tau ; \beta) f(y, \tau, \beta) d y, u \in C^{0}\left(E^{m} \times \bar{I}^{*} \times I\right),
\end{gathered}
$$

where $\Gamma(x, t ; y, \tau ; \beta)$ is the fundamental solution of the operator $\Lambda(x, t, \beta)$.

Lemma 5 generalizes the classic result with $\gamma=0$ to the case with $\gamma \in(0,1)$. It shows also the continuity of $u$ in $E^{m} \times \bar{I}^{*} \times I$. Observe that

$\left|\int_{E^{m}}\left\{\Gamma(x, t ; y, \tau ; \beta)-\Gamma\left(x^{\prime}, t ; y, \tau ; \beta\right)\right\} f(y, \xi, \beta) d y\right| \leq \kappa_{1}\left|x-x^{\prime}\right|(t-\tau)^{-1 / 2}(16)$

for $f \in C^{0}\left(E^{m} \times \bar{I}^{*} \times J\right)$, where $\kappa_{1}$ is a constant.

\section{PROOF OF THEOREM 1.}

Now we are in position to prove Th.1. We limit ourselves to the case of multiple deliveries, i.e. $T<\tau_{22}-\tau_{21}, \tau_{2}^{2}=\tau_{21}+T, \tau_{3}^{2}=\tau_{22}$. The opposite case can be considered in the similar way.

Set 


$$
\begin{aligned}
& Q^{1}=Q_{*}^{1} \cup Q^{1 *}, E^{m} \times I \times I=\cup_{j=0}^{4} Q_{j}^{2}, Q_{j}^{2}=E^{m} \times I \times I_{j}=Q_{j *}^{2} \cup Q_{j}^{2 *}, j=\overline{0,4}, \\
& Q^{3}=Q_{*}^{3} \cup Q^{3 *}, \text { where } \\
& Q_{*}^{1}=\left\{\left(x, t, \tau_{1}\right): x \in E^{m}, 0<t \leq \tau_{1}, \tau_{1} \in I\right\}, \\
& Q^{1 *}=\left\{\left(x, t, \tau_{1}\right): x \in E^{m}, t>\tau_{1}, \tau_{1} \in I\right\}, \\
& Q_{j *}^{2}=\left\{\left(x, t, \tau_{2}\right): x \in E^{m}, 0<t \leq \tau_{2}-\tau_{2}^{j}, \tau_{2} \in I_{j}\right\}, \\
& Q_{j}^{2 *}=\left\{\left(x, t, \tau_{2}\right): x \in E^{m}, t>\tau_{2}-\tau_{2}^{j}, \tau_{2} \in I_{j}\right\}, \\
& Q_{*}^{3}=\left\{\left(x, t, \tau_{1}, \tau_{2}, \tau_{3}\right): x \in E^{m}, 0<t \leq \tau_{3}, \tau_{1} \in \sigma_{1}, \tau_{2} \in \sigma_{2}\left(\tau_{3}\right), \tau_{3} \in \sigma_{3}\right\}, \\
& Q^{3 *}=\left\{\left(x, t, \tau_{1}, \tau_{2}, \tau_{3}\right): x \in E^{m}, t>\tau_{3}, \tau_{1} \in \sigma_{1}, \tau_{2} \in \sigma_{2}\left(\tau_{3}\right), \tau_{3} \in \sigma_{3}\right\} .
\end{aligned}
$$

Let $\tau_{1}=t+\eta_{1}$ and $\tau_{2}=t+\eta_{2}$ be characteristics of the operators $\widehat{D}_{1}$ and $\widehat{D}_{2}$, respectively, and assume that $\tau_{2}=t+\eta_{3}, \tau_{3}=t+\eta_{4}$ mean characteristics of $\widehat{D}_{3}$. Here $\eta_{1}, \eta_{2}, \eta_{3}, \eta_{4}$ denote parameters of characteristics. Letting

$L_{1 s}\left(x, t, t+\eta_{1}\right)=L_{1 s *}\left(x, t, \eta_{1}\right), u_{1 s}\left(x, t, t+\eta_{1}\right)=u_{1 s *}\left(x, t, \eta_{1}\right)$ in $Q_{*}^{1}$,

$L_{1 s}\left(x, \tau_{1}-\eta_{1}, \tau_{1}\right)=L_{1 s}^{*}\left(x, \tau_{1},-\eta_{1}\right)$,

$u_{1 s}\left(x, \tau_{1}-\eta_{1}, \tau_{1}\right)=u_{1 s}^{*}\left(x, \tau_{1},-\eta_{1}\right)$ in $Q^{1 *}$,

$L_{2 k}\left(x, t, t+\eta_{2}\right)=L_{2 k *}\left(x, t, \eta_{2}\right), u_{2 k}\left(x, t, t+\eta_{2}\right)=u_{2 k *}\left(x, t, \eta_{2}\right)$,

$Y_{k}\left(x, t, t+\eta_{2}\right)=Y_{k *}\left(x, t, \eta_{2}\right), X_{k}\left(x, t, t+\eta_{2}\right)=X_{k *}\left(x, t, \eta_{2}\right)$ in $\bigcup_{j=0}^{4} Q_{j *}^{2}$,

$L_{2 k}\left(x, \tau_{2}-\eta_{2}, \tau_{2}\right)=L_{2 k}^{*}\left(x, \tau_{2},-\eta_{2}\right), u_{2 k}\left(x, \tau_{2}-\eta_{2}, \tau_{2}\right)=u_{2 k}^{*}\left(x, \tau_{2},-\eta_{2}\right)$,

$Y_{k}\left(x, \tau_{2}-\eta_{2}, \tau_{2}\right)=Y_{k}^{*}\left(x, \tau_{2},-\eta_{2}\right)$,

$X_{k}\left(x, \tau_{2}-\eta_{2}, \tau_{2}\right)=X_{k}^{*}\left(x, \tau_{2},-\eta_{2}\right)$ in $\bigcup_{j=0}^{4} Q_{j}^{2 *}$,

$L_{3 s k}\left(x, t, \tau_{1}, t+\eta_{3}, t+\eta_{4}\right)=L_{3 s k *}\left(x, t, \tau_{1}, \eta_{3}, \eta_{4}\right), u_{3 s k}\left(x, t, \tau_{1}, t+\eta_{3}, t+\eta_{4}\right)=$ $u_{3 s k *}\left(x, t, \tau_{1}, \eta_{3}, \eta_{4}\right)$ in $Q_{*}^{3}$,

$L_{3 s k}\left(x, \tau_{3}-\eta_{4}, \tau_{1}, \tau_{3}+\eta_{3}-\eta_{4}, \tau_{3}\right)=L_{3 s k}^{*}\left(x, \tau_{3}, \tau_{1},-\eta_{4}, \eta_{3}-\eta_{4}\right)$,

$u_{3 s k}\left(x, \tau_{3}-\eta_{4}, \tau_{1}, \tau_{3}+\eta_{3}-\eta_{4}, \tau_{3}\right)=u_{3 s k}^{*}\left(x, \tau_{3}, \tau_{1},-\eta_{4}, \eta_{3}-\eta_{4}\right)$ in $Q^{3 *}$,

$\tilde{L}_{2 k *}=L_{2 k *}-Y_{k *}, \tilde{L}_{2 k}^{*}=L_{2 k}^{*}-Y_{k}^{*}$

and taking (1)-(12) on the respective characteristics we obtain:

$\left(\partial / \partial t-L_{1 s *}\right) u_{1 s *}=0$ in $Q_{*}^{1}, u_{1 s *}\left(x, 0, \eta_{1}\right)=u_{1 s}^{0}\left(x, \eta_{1}\right)$,

$\left(\partial / \partial \tau_{1}-L_{1 s}^{*}\right) u_{1 s}^{*}=0$ in $Q^{1 *}, u_{1 s}^{*}\left(x, 0,-\eta_{1}\right)=u_{1 s}\left(x,-\eta_{1}, 0\right)$,

$\left(\partial / \partial t-\tilde{L}_{2 k *}\right) u_{2 k *}=X_{k *}$ in $\bigcup_{j=0}^{4} Q_{j *}^{2}, u_{2 k *}\left(x, 0, \eta_{2}\right)=u_{2 k}^{0}\left(x, \eta_{2}\right)$,

$\left(\partial / \partial \tau_{2}-\tilde{L}_{2 k}^{*}\right) u_{2 k}^{*}=X_{k}^{*}$ in $Q_{j}^{2 *}, u_{2 k}^{*}\left(x, \tau_{2}^{j},-\eta_{2}\right)=u_{2 k}\left(x, \tau_{2}^{j}-\eta_{2}, \tau_{2}^{j}\right), j=$ $\overline{0,4}$

$\left(\partial / \partial t-L_{3 s k *}\right) u_{3 s k *}=0$ in $Q_{*}^{3}, u_{3 s k *}\left(x, 0, \tau_{1}, \eta_{3}, \eta_{4}\right)=u_{3 s k}^{0}\left(x, \tau_{1}, \eta_{3}, \eta_{4}\right)$,

$\left(\partial / \partial \tau_{3}-L_{3 s k}^{*}\right) u_{3 s k}^{*}=0$ in $Q^{3 *}, u_{3 s k}^{*}\left(x, 0, \tau_{1},-\eta_{4}, \eta_{3}-\eta_{4}\right)=$

$u_{3 s k}\left(x,-\eta_{4}, \tau_{1}, \eta_{3}-\eta_{4}, 0\right)$.

By virtue of $\left(H_{3}\right)-\left(H_{5}\right)$ the operators $L_{1 s *}, L_{2 k *}, L_{3 s k *}, L_{1 s}^{*}, L_{2 k}^{*}, L_{3 s k}^{*}$ and initial distributions $u_{1 s *}\left(x, 0, \eta_{1}\right), u_{2 k *}\left(x, 0, \eta_{2}\right), u_{3 s k *}\left(x, 0, \tau_{1}, \eta_{3}, \eta_{4}\right)$ satisfy all the conditions of Lemma 5 . If $Y_{k *}, Y_{k}^{*}, u_{1 s}\left(x,-\eta_{1}, 0\right), u_{2 k}\left(x, \tau_{2}^{j}-\right.$ $\left.\eta_{2}, \tau_{2}^{j}\right), u_{3 s k}\left(x,-\eta_{4}, \tau_{1}, \eta_{3}-\eta_{4}, 0\right)$ and $X_{k *}, X_{k}^{*}$ are known and satisfy all the conditions of Lemma 5 , then system (17) degenerates into separate problems for $u_{1 s *}, u_{1 s}^{*}, u_{2 k *}, u_{2 k}^{*}, u_{3 s k *}, u_{3 s k}^{*}$, respectively, of type (14). Then denoting by

$$
\Gamma_{1 s *}\left(x, t ; y, \xi ; \eta_{1}\right), \Gamma_{1 s}^{*}\left(x, \tau_{1} ; y, \xi ;-\eta_{1}\right), \Gamma_{2 k j *}\left(x, t ; y, \xi ; \eta_{2}\right), \Gamma_{2 k j}^{*}\left(x, \tau_{2} ; y,\right.
$$


$\left.\xi ;-\eta_{2}\right), \Gamma_{3 s k *}\left(x, t ; y, \xi ; \tau_{1}, \eta_{3}, \eta_{4}\right), \Gamma_{3 s k}^{*}\left(x, \tau_{3} ; y, \xi ; \tau_{1},-\eta_{4}, \eta_{3}-\eta_{4}\right)$ the fundamental solutions of operators

$\partial / \partial t-L_{1 s *}, \partial / \partial \tau_{1}-L_{1 s}^{*}, \partial / \partial t-\tilde{L}_{2 k *}, \partial / \partial \tau_{2}-\tilde{L}_{2 k}^{*}, \partial / \partial t-L_{3 s k *}, \partial / \partial \tau_{3}-$ $L_{3 s k}^{*}$

in $Q_{*}^{1}, Q^{1 *}, Q_{j *}^{2}, Q_{j}^{2 *}, Q_{*}^{3}, Q^{3 *}$, respectively, and, applying general formula (15) to $u_{1 s *}, u_{2 k *}, u_{3 s k *}, u_{1 s}^{*}, u_{2 k}^{*}, u_{3 s k}^{*}$, from (17) we obtain the system

$u_{1 s *}\left(x, t, \eta_{1}\right)=\int_{E^{m}} \Gamma_{1 s *}\left(x, t ; y, 0 ; \eta_{1}\right) u_{1 s}^{0}\left(y, \eta_{1}\right) d y$ in $Q_{*}^{1}$,

$u_{1 s}^{*}\left(x, \tau_{1},-\eta_{1}\right)=\int_{E^{m}} \Gamma_{1 s}^{*}\left(x, \tau_{1} ; y, 0 ;-\eta_{1}\right) u_{1 s}\left(y,-\eta_{1}, 0\right) d y$ in $Q^{1 *}$,

$u_{2 k *}\left(x, t, \eta_{2}\right)=\int_{E^{m}} \Gamma_{2 k j *}\left(x, t ; y, 0 ; \eta_{2}\right) u_{2 k}^{0}\left(y, \eta_{2}\right) d y+$

$\int_{0}^{t} d \xi \int_{E^{m}} \Gamma_{2 k j *}\left(x, t ; y, \xi ; \eta_{2}\right) X_{k *}\left(y, \xi, \eta_{2}\right) d y$ in $Q_{j *}^{2}$,

$u_{2 k}^{*}\left(x, \tau_{2},-\eta_{2}\right)=\int_{E^{m}} \Gamma_{2 k j}^{*}\left(x, \tau_{2} ; y, \tau_{2}^{j} ;-\eta_{2}\right) u_{2 k}\left(y, \tau_{2}^{j}-\eta_{2}, \tau_{2}^{j}\right) d y+$

$\int_{\tau_{2}^{j}}^{\tau_{2}} d \xi \int_{E^{m}} \Gamma_{2 k j}^{*}\left(x, \tau_{2} ; y, \xi ;-\eta_{2}\right) X_{k}^{*}\left(y, \xi,-\eta_{2}\right) d y$ in $Q_{j}^{2 *}$,

$u_{3 s k *}\left(x, t, \tau_{1}, \eta_{3}, \eta_{4}\right)=\int_{E^{m}} \Gamma_{3 s k *}\left(x, t ; y, 0 ; \tau_{1}, \eta_{3}, \eta_{4}\right) u_{3 s k}^{0}\left(y, \tau_{1}, \eta_{3}, \eta_{4}\right) d y$

in $Q_{*}^{3}$,

$u_{3 s k}^{*}\left(x, \tau_{3}, \tau_{1},-\eta_{4}, \eta_{3}-\eta_{4}\right)=$

$\int_{E^{m}} \Gamma_{3 s k}^{*}\left(x, \tau_{3} ; y, 0 ; \tau_{1},-\eta_{4}, \eta_{3}-\eta_{4}\right) u_{3 s k}\left(y,-\eta_{4}, \tau_{1}, \eta_{3}-\eta_{4}, 0\right) d y$ in $Q^{3 *}$,

which by (4) and (6-9) can be written as follows:

$u_{1 s}\left(x, t, \tau_{1}\right)=\int_{E^{m}} \Gamma_{1 s *}\left(x, t ; y, 0 ; \tau_{1}-t\right) u_{1 s}^{0}\left(y, \tau_{1}-t\right) d y$ in $Q_{*}^{1}$,

$u_{1 s}\left(x, t, \tau_{1}\right)=\int_{E^{m}} \Gamma_{1 s}^{*}\left(x, \tau_{1} ; y, 0 ; t-\tau_{1}\right) u_{1 s}\left(y, t-\tau_{1}, 0\right) d y$ in $Q^{1 *}$,

$u_{2 k}\left(x, t, \tau_{2}\right)=$

$\int_{E^{m}} \Gamma_{2 k j *}\left(x, t ; y, 0 ; \tau_{2}-t\right) u_{2 k}^{0}\left(y, \tau_{2}-t\right) d y$ in $Q_{j *}^{2}$ for $j=0,1,4$,

$u_{2 k}\left(x, t, \tau_{2}\right)=\int_{E^{m}} \Gamma_{2 k j *}\left(x, t ; y, 0 ; \tau_{2}-t\right) u_{2 k}^{0}\left(y, \tau_{2}-t\right) d y$

$+\sum_{s} \int_{0}^{t} d \xi \int_{E^{m}} d y \Gamma_{2 k j *}\left(x, t ; y, \xi ; \tau_{2}-t\right)$

$\int_{\sigma_{1}} u_{3 s k}\left(y, \xi, \tau_{1}, \xi+\tau_{2}-t, T\right) d \tau_{1}$ in $Q_{j *}^{2}$ for $j=2,3$,

$u_{2 k}\left(x, t, \tau_{2}\right)=\int_{E^{m}} \Gamma_{2 k j}^{*}\left(x, \tau_{2} ; y, \tau_{2}^{j} ; t-\tau_{2}\right) u_{2 k}\left(y, \tau_{2}^{j}+t-\tau_{2}, \tau_{2}^{j}\right) d y$ in $Q_{j}^{2 *}$

for $j=0,1,4$,

$u_{2 k}\left(x, t, \tau_{2}\right)=\int_{E^{m}} \Gamma_{2 k j}^{*}\left(x, \tau_{2} ; y, \tau_{2}^{j} ; t-\tau_{2}\right) u_{2 k}\left(y, \tau_{2}^{j}+t-\tau_{2}, \tau_{2}^{j}\right) d y$

$+\sum_{s} \int_{\tau_{2}^{j}}^{\tau_{2}} d \xi \int_{E^{m}} d y \Gamma_{2 k j}^{*}\left(x, \tau_{2} ; y, \xi ; t-\tau_{2}\right)$

$\int_{\sigma_{1}} u_{3 s k}\left(y, \xi+t-\tau_{2}, \tau_{1}, \xi, T\right) d \tau_{1}$ in $Q_{j}^{2 *}$ for $j=2,3$, 


$$
\begin{aligned}
& u_{3 s k}\left(x, t, \tau_{1}, \tau_{2}, \tau_{3}\right)= \\
& \int_{E^{m}} \Gamma_{3 s k *}\left(x, t ; y, 0 ; \tau_{1}, \tau_{2}-t, \tau_{3}-t\right) u_{3 s k}^{0}\left(y, \tau_{1}, \tau_{2}-t, \tau_{3}-t\right) d y \text { in } Q_{*}^{3}, \\
& u_{3 s k}\left(x, t, \tau_{1}, \tau_{2}, \tau_{3}\right)= \\
& \int_{E^{m}} \Gamma_{3 s k}^{*}\left(x, \tau_{3} ; y, 0 ; \tau_{1}, t-\tau_{3}, \tau_{2}-\tau_{3}\right) \\
& u_{3 s k}\left(y, t-\tau_{3}, \tau_{1}, \tau_{2}-\tau_{3}, 0\right) d y \text { in } Q^{3 *}, \\
& u_{3 s k}\left(x, t, \tau_{1}, \tau_{2}, 0\right)=p_{s k}\left(x, t, \tau_{1}, \tau_{2}\right) u_{1 s}\left(x, t, \tau_{1}\right) u_{2 k}\left(x, t, \tau_{2}\right) / n_{1}(x, t), \\
& n_{1}=\sum_{i} \int_{\sigma_{1}} u_{1 i}\left(x, t, \tau_{1}\right) d \tau_{1}, \\
& u_{1 s}(x, t, 0)=\sum_{i k} \int_{\sigma} b_{1 i k}\left(x, t, \tau_{1}, \tau_{2}, n(x, t-T)\right) u_{3 i k}\left(x, t, \tau_{1}, \tau_{2}, T\right) \omega_{i k}^{s} d \sigma, \\
& u_{2 k}(x, t, 0)=\sum_{s i} \int_{\sigma} b_{2 s i}\left(x, t, \tau_{1}, \tau_{2}, n(x, t-T)\right) u_{3 s i}\left(x, t, \tau_{1}, \tau_{2}, T\right) \omega_{s i}^{k} d \sigma . \\
& \sum_{s} \int_{I} u_{1 s} d \tau_{1}+\sum_{k} \int_{I} u_{2 k} d \tau_{2}+ \\
& \sum_{s k} \int_{\sigma_{3}} d \tau_{3} \int_{\sigma_{2}\left(\tau_{3}\right)} d \tau_{\sigma_{1}} \int_{\sigma_{1}} u_{3 s k} d \tau_{1} \text { in } E^{m} \times I, \\
& E^{m} \times[-T, 0],
\end{aligned}
$$

We must add to (22) and (23) the continuity condition $\left[\left.u_{2 k}\right|_{\tau_{2}=\tau_{2}^{j}}\right]=0, j=$ $\overline{1,4}$.

Now we will prove that (18)-(29) represent the solution of (1)-(13). Consider system (18)-(29) moving along the axis $t$ by the step of size $T$. Since $L_{1 s *}, L_{2 k *}$ in $Q_{0 *}^{2} \cup Q_{4 *}^{2}$, and $L_{3 s k *}$ satisfy the conditions of Lemma 5, formulas (18), (20) for $j=0$, and (24) express strictly positive functions $u_{1 s}, u_{2 k}$ and $u_{3 s k}$ in $Q_{*}^{1}, Q_{0 *}^{2} \cup Q_{4 *}^{2}$ and $Q_{*}^{3}$, respectively. Hence $\left.n_{1}\right|_{\text {supp }} \mathrm{p}_{\mathrm{sk}}\left(\cdot, \mathrm{t}, \tau_{1}, \tau_{2}\right) \geq \tilde{n}_{1}$, and by virtue of $\left(H_{1}\right)$ we observe that

$p_{s k}\left(x, t, \tau_{1}, \tau_{2}\right) u_{1 s}\left(x, t, \tau_{1}\right) / n_{1}(x, t) \in C^{0}\left(E^{m} \times\left[0, \tau_{11}\right] \times \bar{\sigma}_{1} \times \bar{\sigma}_{2}(0)\right)$, where $\tilde{n}_{1}$ is a positive constant, while from (24) by (16) it follows that

$\left|u_{3 s k}\left(x, t, \tau_{1}, \tau_{2}, \tau_{3}\right)-u_{3 s k}\left(y, t, \tau_{1}, \tau_{2}, \tau_{3}\right)\right| \leq \kappa_{1}|x-y| t^{-1 / 2}$ in $Q_{*}^{3}$ with $\kappa_{1}$ a constant.

Let $t \in[0, T]$ and assume $\omega_{1}=E^{m} \times[0, T] \times \bar{I}$. By means of (27), (28), (29), $\left(H_{2}\right),\left(H_{5}\right)$ and due to the continuity of $u_{3 s k}$ (see Lemma 5 ) we obtain continuous $u_{1 s}(x, t, 0)$ and $u_{2 k}(x, t, 0) \forall(x, t) \in E^{m} \times[0, T]$. Now from (19) and (22) for $j=0$ we get continuous $u_{1 s}$ and $u_{2 k}$ in $Q^{1 *} \cap \omega_{1}$ and $Q_{0}^{2 *} \cap \omega_{1}$, respectively. Then by $\left(H_{1}\right)$,

$$
Y_{k}\left(x, t, \tau_{2}\right)=\left\{\begin{array}{l}
0, \tau_{2} \notin \sigma_{2}(0) \\
p_{k}, \tau_{2} \in \sigma_{2}(0),
\end{array}\right.
$$

and $\left(H_{4}\right)$ shows that $\tilde{L}_{2 k *}$ and $\tilde{L}_{2 k}^{*}$ satisfy all the conditions of Lemma 5 in $\left(Q_{1 *}^{2} \cup Q_{2 *}^{2}\right) \cap \omega_{1}$ and $\left(Q_{1}^{2 *} \cup Q_{2}^{2 *}\right) \cap \omega_{1}$, respectively. Then (20) and (22) for $j=1$ yield $u_{2 k}$ in $\left(Q_{1 *}^{2} \cup Q_{1}^{2 *}\right) \cap \omega_{1}$, while from (21) and (23) by (24) and (30) 
we get $u_{2 k}$ in $\left(Q_{2 *}^{2} \cup Q_{3 *}^{2}\right) \cap \omega_{1}$ and $\left(Q_{2}^{2 *} \cup Q_{3}^{2 *}\right) \cap \omega_{1}$, respectively. Eq. (22) for $j=4$ gives $u_{2 k}$ in $Q_{4}^{2 *} \cap \omega_{1}$. Recalling the maximum principle and using $\left(H_{5}\right)$ and $(29)$ we prove the continuity of $n(x, t)$.

Let $t \in(T, 2 T]$ and assume $\omega_{2}=E^{m} \times[T, 2 T] \times \bar{I}$. Knowing $u_{1 s}$ and $u_{2 k}$ for $t \in[0, T]$, by $(26),\left(H_{1}\right)$ and because of $\left.n_{1}\right|_{\text {supp }} \mathrm{p}_{\mathrm{k}}\left(\cdot, \mathrm{t}, \tau_{2}\right) \geq \tilde{n}_{1}$ we get continuous $u_{3 s k}\left(x, t, \tau_{1}, \tau_{2}, 0\right)$ for $t \in\left(\tau_{3}, T\right]$, then by $(25)$ we obtain $u_{3 s k}\left(x, t, \tau_{1}, \tau_{2}, \tau_{3}\right)$ for $t \in\left(\tau_{3}, \tau_{3}+T\right]$. From (27) and (28), by using $\left(H_{2}\right)$ and known continuous $n(x, t),\left.u_{3 s k}\right|_{\tau_{3}=T}$, we get continuous $\left.u_{1 s}\right|_{\tau_{1}=0}$ and $\left.u_{2 k}\right|_{\tau_{2}=0}$, too. Then by virtue of (19) with known $\left.u_{1 s}\right|_{\tau_{1}=0}$ and by (22) with known $\left.u_{2 k}\right|_{\tau_{2}=0}$ we obtain $u_{1 s}$ and $u_{2 k}$ in $Q^{1 *} \cap \omega_{2}$ and $Q_{0}^{2 *} \cap \omega_{2}$, respectively. Now by (22) for $j=1$, (23) for $j=2,(21)$ for $j=2,(23)$ for $j=3,(22)$ for $j=4$, and (20) for $j=4$ we construct $u_{2 k}$ in $\left(\bigcup_{j=1}^{4} Q_{j}^{2 *} \cup Q_{2 *}^{2}\right) \cap \omega_{2}$.

Proceeding our reasoning we obtain $u_{1 s}, u_{2 k}$ and $u_{3 s k}$ for $t \in\left[2 T, t^{*}\right]$. Restrictions (13) ensure the continuity of $u_{1 s}, u_{2 k}, u_{3 s k}$ across the lines $t=$ $\tau_{1}, t=\tau_{2}, t=\tau_{3}$, respectively. So Th. 1 is proved.

\section{REFERENCES}

[1] A. Friedman, Differential equations with partial derivatives of the parabolic type, Mir, Moscow, 1968 (in Russian).

[2] M.G. Garoni, J.L. Menaldi, Green functions for second order parabolic integrodifferential problems, Pitman Research Notes in Mathematics, Series 275 ISSN 02693677, New York, 1992.

[3] O.A. Ladyzhenskaya, V.A. Solonnikov, N.N. Ural'tseva, Linear and Quasilinear equations of parabolic type, Nauka, Moscow, 1967 (in Russian).

[4] V. Skakauskas, An evolution model of an autosomal polylocal polyallelic diploid population taking into account crossing-over and gestation period, Lith. math. J., 34, No. 3 (1994), P. 288-300.

[5] V. Skakauskas, A mathematical analysis of an age-sex-space-structured population dynamics model with random mating and females' pregnancy, Informatica (Lith. Acad. of Sci.) ISSN 068-1952, 9, No. 3 (1998) (to appear).

[6] V. Skakauskas, A mathematical analysis of an age-space-structured autosomal diploid population dynamics model with random mating and females' pregnancy, Lith. math. J. 38 No. 4 (1998) (to appear).

[7] Yu. Svirezhev, V.P. Passekov, Fundamentals of mathematical evolutionary genetics. Kluwer academic publishers, Dordrecht, 1990. 